\title{
REVIEW ARTICLE \\ Local and systemic effects of IL-17 in joint inflammation: a historical perspective from discovery to targeting
}

\author{
Pierre Miossec (iD)
}

\begin{abstract}
The role of IL-17 in many inflammatory and autoimmune diseases is now well established, with three currently registered anti-IL-17targeted therapies. This story has taken place over a period of 20 years and led to the demonstration that a $\mathrm{T}$ cell product could regulate, and often amplify, the inflammatory response. The first results described the contribution of IL-17 to local features in arthritis. Then, understanding was extended to its systemic effects, with a focus on cardiovascular aspects. This review provides a historical perspective of these discoveries focused on arthritis, which started in 1995, followed 10 years later by the description of Th17 cells. Today, IL-17 inhibitors for three chronic inflammatory diseases have been registered. More options are now being tested in ongoing and future clinical trials. Inhibitors of IL-17 family members and Th17 cells ranging from antibodies to small molecules are under active development. The identification of patients with IL-17-driven disease is a key target for the improved selection of patients expected to have a strongly positive response.
\end{abstract}

Keywords: Interleukin-17; Th17 cells; Inflammation; Arthritis, targeting

Cellular \& Molecular Immunology (2021) 18:860-865; https://doi.org/10.1038/s41423-021-00644-5

\section{INTRODUCTION}

Human IL-17, a proinflammatory cytokine identified in 1995 as a product of activated T cells, ${ }^{1}$ is involved in the pathogenesis of rheumatoid arthritis (RA) $)^{2}$ and many other autoimmune and inflammatory diseases. In 2015, the first inhibitor of IL-17 was approved for the treatment of one such disease. ${ }^{3}$ Over the past 20 years, research has shown the key inflammatory properties of IL17 in vitro and in vivo first in arthritis and then in many other conditions. Key progress came from the identification of a new subset of T cells that produce IL-17, thus named Th17 cells, which are distinct from the Th1 and Th2 subsets. ${ }^{4,5}$ IL-17 is now the target of various therapeutic options approved for psoriasis, psoriatic arthritis, and ankylosing spondylitis. ${ }^{6}$

This review is focused on the contribution of IL-17 to the local and systemic manifestations of arthritis. It first describes the discovery of IL-17, its receptors, and its biological effects on various local targets. The second section outlines the key contribution of cell-cell interactions in the increased production of IL-17 at the local site of inflammation. The following section discusses the systemic effects of IL-17. Finally, different options for targeting IL-17 are presented.

\section{DISCOVERY AND LOCAL EFFECTS OF IL-17}

IL-17 was discovered in 1993 as the product of a gene isolated first in rodent cells as cytotoxic T lymphocyte associated-antigen 8 $(\mathrm{CTLA}-8)^{7}$ and then in human activated T cells. ${ }^{1}$ Its activity in humans was first demonstrated through the use of several cell types, including human foreskin fibroblasts ${ }^{1}$ and normal and RA synoviocytes, in which CTLA-8 induced the production of IL-6 and
IL-8. ${ }^{2,8}$ Analysis of its structure and functions showed that this protein was a new molecule with cytokine characteristics that was then named IL-17. ${ }^{9}$ At the same time, the receptor for IL-17 was identified and again shown to be a new molecule differing from already known cytokine receptors. ${ }^{1}$ Further analysis of protein sequences identified other proteins showing sequence similarity with IL-17. Based on this homology, the concept of the IL-17 family was introduced, and IL-17 was renamed IL-17A. ${ }^{10}$ Other members range from high to low in terms of their sequence homology with IL-17A include and IL-17F (with 50\% homology), IL-17B, IL-17C, IL$17 \mathrm{D}$, and IL-17E, which shows the lowest sequence homology at $19 \%$. IL-17A and $F$ have rather similar activities, but IL-17F is less potent. ${ }^{11}$ Studies with synovium samples showed that IL-17Fproducing cells were more abundant that those producing IL-17A, with more IL-17F produced than IL-17A. ${ }^{12}$ Whereas other IL-17 family members have proinflammatory effects, IL-17E, also known as IL-25, has anti-inflammatory effects and reduces the effects of IL-17A and IL-17F. ${ }^{11}$ In synoviocytes, IL-17 was found to first induce the production of proinflammatory cytokines, such as IL-6 and IL-8, with IL- 6 and IL-8 production plateauing after 5 days when IL-25 starts to be produced. ${ }^{13}$

The key local effects of IL-17A in arthritis were identified by these early studies (Fig. 1). They first showed that IL-17A used alone had relatively modest effects on different cell types compared to the activities of the key inflammatory cytokines TNF and IL-1. However, it was quickly shown that when combined with TNF or IL-1, IL-17A massively increased the production of IL-6 and IL-8. ${ }^{8}$ From these early studies, it was concluded that IL-17 increases the level of inflammation, as reflected by the production of IL-6. It simultaneously activates neutrophils through its

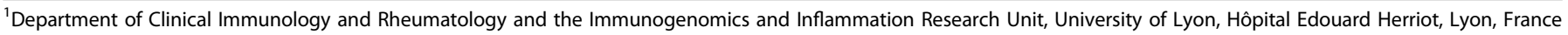
Correspondence: Pierre Miossec (pierre.miossec@univ-lyon1.fr)

Received: 27 October 2020 Accepted: 18 January 2021

Published online: 10 March 2021 


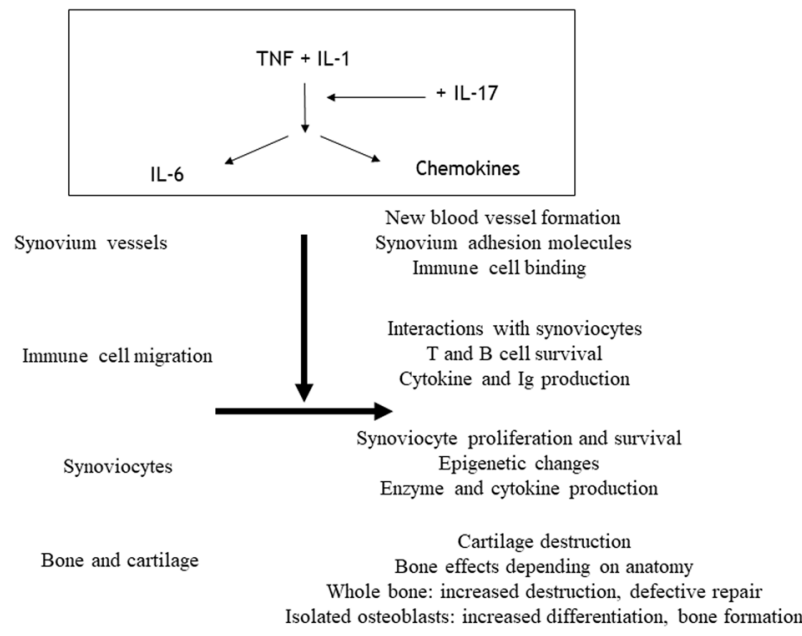

Fig. 1 Local effects of IL-17 in combination with other proinflammatory cytokines. The key monocyte-derived cytokines TNF and IL-1 act on stromal cells to induce the production of $\mathrm{IL}-6$ and chemokines. This effect is amplified by IL-17, often through synergistic interactions. The resulting effects act on vessels, inducing pathogenic cell migration. This induces the activation of local stromal cells and migrated pathogenic cells and increases their survival. Consequently, these effects result in changes to the matrix structure and metabolism. The net result depends on the tissue involved and its anatomic localization. In whole bone, where osteoclasts are in contact with osteoblasts, these cytokines induce bone destruction. In tendons and ligaments, which lack osteoclasts, the same cytokines induce stromal cell differentiation into osteoblasts, resulting in ectopic bone formation. This figure is focused on differences in the various presentations of arthritis. Most of the effects can be applied to other chronic and acute conditions

substantial effects on the production of IL-8, a key chemokine for neutrophils during acute inflammation.

The first clinical application of IL-17 was in RA, with the demonstration of increased levels of IL-17 in RA synovial fluid. ${ }^{14} \mathrm{At}$ the same time, the presence of bioactive IL-17 in the supernatants of cultures from samples of the inflamed RA synovium was shown. ${ }^{15}$ An antibody targeting $\mathrm{LL}-17 \mathrm{~A}$ reduced the production of IL- 6 by synoviocytes induced by these supernatants by twothirds. ${ }^{15}$ This confirmed that IL-17A was present and functional. There was an important difference in activity between its modest effects on synoviocytes when used alone and the more substantial effects inferred when its function was inhibited in supernatants. This discrepancy indicated that IL-17 in fact interacts with many other cytokines, including TNF and IL-1 but also GM-CSF and IFNY, often in a synergistic manner. ${ }^{16}$ Staining of synovium samples showed that IL-17-positive cells were indeed present at low numbers and the probable source of bioactive IL-17. ${ }^{15}$ Further studies of RA synovium sections stained with an anti-IL-17 antibody showed that IL-17-positive cells had a large size with a large cytoplasmic area and remote nucleus, resembling plasma cells. ${ }^{17}$ In vitro studies with activated PBMC showed that cells positive for IL-17 by intracellular staining were indeed CD4 T cells that had lost TCR and CD3 during activation and differentiation into cytokine-producing cells, most likely becoming insensitive to an antigen-driven activation at that stage. ${ }^{17}$

The discovery of receptors for IL-17 evolved in parallel. Following the identification of the first receptor in 1995, sequence studies similar to those carried out with the IL-17 protein were performed with IL-17R. The first described receptor for IL-17 was also found to be the first member of a new family of cytokine receptors. ${ }^{1}$ It was then discovered that the receptor for IL-17A consists of two chains: the first chain, IL-17-R, which was renamed IL-17RA, and a new chain, IL-17RC. ${ }^{18}$ The IL-17RA/RC complex was shown to be the receptor complex for IL-17A and IL-17F. ${ }^{18,19}$
IL-17E/IL-25 uses the IL-17RA chain but does so in association with the IL-17RB receptor chain. ${ }^{20}$ This could allow both IL-17A and IL$17 F$ to compete with IL-17E/IL-25 for the IL-17RA chain. Such use of two different receptors could explain why IL-17A and IL-17F, compared to IL-17E/IL-25, have opposite pro- and antiinflammatory effects, respectively. IL-17RA is essential for IL-17A, IL-17F, and IL-17E/IL-25 signaling ${ }^{19}$ and may act as a shared receptor for the IL-17 family, as observed for gp130 with the IL-6 cytokine family. ${ }^{21}$ Compared to its affinity for IL-17A, which is high, IL-17RA has a 100-fold weaker affinity for IL-17F and much weaker affinity for IL-17E/IL-25. ${ }^{19,21}$

Discoveries related to the IL-17 proteins and receptors have led to a better understanding of the interactions between the proinflammatory cytokines TNF and IL-1 with IL-17A or IL-17F. To obtain a synergistic effect with IL-17 and TNF in combination, a particular order is needed; IL-17 acts first, increasing the expression of TNF receptors, specifically type II TNFR. ${ }^{22}$ This increased expression of type II TNFR then amplifies the response to TNF, as reflected by a massive increase in the downstream production of cytokines including IL-6, IL-8, G- and GM-CSF, LIF, and CCL-20. ${ }^{16,23}$ This synergy is not observed if TNF acts first followed by IL-17. ${ }^{22}$ This amplification can also occur through an increase in mRNA half-life, resulting in increased and more sustained protein production. The II-17 receptor-associated adaptor molecule Act1 was found to have a direct effect on mRNA stabilization, leading to increased signaling and inflammation. ${ }^{24}$ Recent results have further demonstrated that these effects on amplification act as a cascade at the level of transcription factor activation. ${ }^{25,26}$

The function of $\mathrm{IL}-17$ is regulated at the local and systemic levels by the positive and negative effects of many soluble factors. TNF, IL-1, GM-CSF, and IFNY have additive or synergistic effects with IL-17A, whereas IL-25/IL-17E, anti-IL-17 autoantibodies and soluble IL-17R inhibit IL-17A function. ${ }^{27}$ As an example, compared to those without anti-IL-17 autoantibodies, RA patients with antiIL-17 autoantibodies exhibit decreased joint destruction, suggesting the protective effect of these antibodies. ${ }^{28}$ Together, these results indicate the importance of considering the bioactivity of IL17 and not just its protein concentration.

Extensive profiling of different cell types to understand the net effect of these cytokines acting together at an inflammatory site has shown that these cytokines act in the defined order described above. The synergy between IL-17 and TNF occurs only when IL-17 acts before TNF and not when TNF acts first. ${ }^{22}$ Various options related to gene regulation to overcome this have been observed. One such option is amplifying the responses of genes already induced by each cytokine alone. Another option is the induction of genes induced only when a combination of cytokines is present. For example, in synoviocytes, if IL-17A alone induces 100 genes and TNF alone 1000 genes, in combination, they induce 10,000 genes. $^{12}$ This concept is important when applied to clinical conditions, such as arthritis in which there is no need for large amounts of cytokines because of these synergistic interactions. Locally, this is further amplified by cell-cell interactions, specifically those between T cells and stromal cells, such as synoviocytes. ${ }^{29}$

\section{ROLE OF CELL-CELL INTERACTIONS IN TH17 CELL ACTIVATION AND IL-17 PRODUCTION}

Interest in the IL-17 field greatly increased when it was shown that IL-17 is produced by a new cell subset that was first named T helper cells producing IL-17 and later renamed Th17 cells. ${ }^{4,5}$ Human IL-17 was first described in 1995, and the Th17 cell type was described in only 2005, indicating that it took 10 years to move from a cell product to the cell type responsible for its production. The first indications that IL-17 is produced by T cells were found early using $T$ cell clones from the blood of patients with allergic contact dermatitis ${ }^{30}$ and the synovium of arthritis patients. ${ }^{31}$ In experiments with synovial T cells, IL-17 could be produced alone or with IFNy but 
not with IL-4. ${ }^{31}$ It was later shown that pathogenic Th17 cells express and produce both IL-17 and IFN $\gamma$. Full demonstration that the Th17 pathway is distinct came from the identification of the lineage-specific transcription factors Roryt/Rorc, which differ from Tbet and Gata3, markers of the Th1 and Th2 lineages, respectively. ${ }^{32}$ Additional studies showed that many other cell types, including CD8 + T cells, $\gamma \delta T$ cells, invariant natural killer T cells, and innate lymphoid cells, produce IL-17. ${ }^{33}$ Furthermore, mast cells and neutrophils in RA synovial sections stained positive for IL-17, indicating that they engulf but do not produce IL-17. ${ }^{34,35}$

Conditions for the induction of Th17 differentiation were established first when the role of the monocyte-derived inflammatory cytokines IL- 1 and IL-6 as early drivers was shown. ${ }^{36}$ Understanding of the specificity of the new Th17 pathway led to the discovery of IL-23, which is produced by dendritic cells. ${ }^{37-39}$ In the context of a mouse disease model, IL-23 was found to have a key effect in the induction of Th17 pathogenicity. ${ }^{40}$ As the contribution of the Th17 pathway is further amplified in many diseases, the role of defective regulation was established by showing the importance of the strict balance between Th17 and regulatory $\mathrm{T}$ cells. ${ }^{41}$ However, this balance is highly sensitive to changes, and because of their plasticity, Th17 cells have the capacity to convert into regulatory $T$ cells. ${ }^{42}$

In regard to clinical understanding of the role of Th cell subsets in disease, a key starting event is the migration of disease-associated $T$ cells (specifically in Fig. 1, synovium-associated $T$ cells) to the site of inflammation (Fig. 1). ${ }^{29}$ After migration, the T cells interact with local stromal cells (specifically in Fig. 1, synoviocytes). ${ }^{29}$ Diseases like RA are associated with the massive proliferation, defective apoptosis, and increased invasiveness of synoviocytes.

In vitro models have been established to study these interactions. ${ }^{43}$ Contact between circulating human mononuclear cells and RA synoviocytes alone is sufficient to induce IL-6 and IL-8 production, mostly by synoviocytes. ${ }^{43}$ This effect is strongly reduced in a Transwell system that prevents cell contact. ${ }^{43}$ In contrast, the induction of IL-17 production needs both TCR activation and cell contact, the combination of which induces a massive increase in the production of IL-17. ${ }^{43}$ The results obtained with psoriasis skin-derived fibroblasts and synoviocytes are similar, except for a key difference in the contribution of monocytes. ${ }^{44}$ In both cases, such cytokine production is inhibited, as shown by the $50 \%$ reduction with an anti-podoplanin antibody. This indicates the contribution of other molecules.

Although the combination of these cytokines with IL-17 often leads to amplification, this is not always the case. One example is the opposite effects of these cytokines on bone cells with their direct application to different arthritis diseases. The net result depends on the structure of the tissue and its anatomic localization. On whole-bone explants, where osteoclasts are in contact with osteoblasts, these cytokines induce bone destruction. ${ }^{14,45}$ Inhibition of IL-17 protects against bone destruction, and the combination of inhibitors of IL-17, IL-1, and TNF is more potent than the individual inhibitors alone ${ }^{46}$. Conversely, in tendons and ligaments, which lack osteoclasts, the same cytokines induce ectopic bone formation from stromal cell differentiation into osteoblasts. ${ }^{47}$ The effects observed in whole bone are found in RA and psoriatic arthritis, in which destruction predominates. The other effects are found in ankylosing spondylitis in the spine, in which inflammation leads to ectopic bone formation with the ossification of ligaments. This explains why the same IL-17 inhibitors are used in psoriatic arthritis, in which bone destruction is dominant in the peripheral joints, and in ankylosing spondylitis, which is characterized by spinal bone formation.

\section{SYSTEMIC EFFECTS OF IL-17}

These first studies investigated the effects of IL-17 on different targets involved in the local manifestations of diseases (Fig. 1). The studies on these local effects have been based on targeting IL-1, TNF, IL-6, and now IL-17 through biological therapies. ${ }^{6,48}$ More recently, the same chronic diseases were discovered to share an increased risk of cardiovascular events. ${ }^{49}$

As for other inflammatory cytokines, studies on IL-17 have focused on these new but rather forgotten targets. ${ }^{50}$ Regarding the local features described above, it is important to consider that cytokines interact as a team in blood and tissues (Fig. 2).

Effects on vessels cells are obviously of major importance. On endothelial cells, the inflammatory effects of IL-17 are the same with IL-6 and IL-8 induction. ${ }^{51}$ Here, synergy between TNF or IL-1 and IL-17 against massively increases these effects. Cell specificity is linked to specific vascular functions. For instance, TNF and IL-17 in combination strongly increase procoagulation activity in endothelial cells, with a synergistic increase in tissue factor, leading to activation of the coagulation cascade. ${ }^{51}$ At the same time, the same conditions induce the inhibition of protective antithrombotic factors, such as thrombomodulin. ${ }^{51}$ The importance of these cytokines in clinical thrombosis is further supported by the demonstration of peak IL-17 bioactivity in the circulation of patients with acute myocardial infarction. ${ }^{52}$

The liver is another important target of systemic inflammation. On hepatocytes, the combination of TNF and IL-17 induces the massive release of IL- $6 .^{22}$ In turn, IL- 6 induces a large increase in $C$ reactive protein (CRP). Such an increase in CRP was fully inhibited in vitro and in vivo with an anti-IL-6R Ab. ${ }^{22}$ This confirms that IL-6 is a key molecule in the liver inflammation cascade, induced by TNF and IL17.22 In turn, CRP is a key marker for an increased risk of cardiovascular events. ${ }^{53}$ However, IL-6 is not the only critical cytokine in liver inflammation. Again, the combination of IL-17 and TNF induces IL- 8 secretion by hepatocytes, but this was not inhibited by an antibody against IL-6R. ${ }^{22}$ The same conclusion

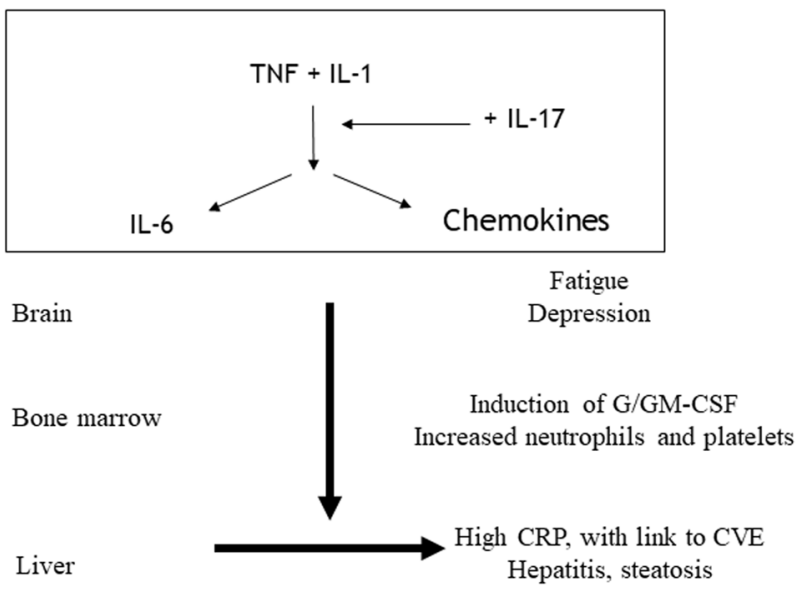

Vessels

Procoagulation

Platelet adhesion

Thrombosis, emboli

\section{Muscles \\ Stiffness \\ Muscle loss}

Fig. 2 Systemic effects of $\mathrm{IL}-17$ in combination with other proinflammatory cytokines. Regarding local effects, the key monocyte-derived cytokines TNF and IL-1 act on stromal cells to induce the production of IL- 6 and chemokines. This effect is amplified by IL-17, often through synergistic interactions. These effects act on the brain, bone marrow, vessels, liver, and muscles. This figure depicts the overall clinical picture for many chronic and acute conditions, including the cytokine storm in COVID-19 


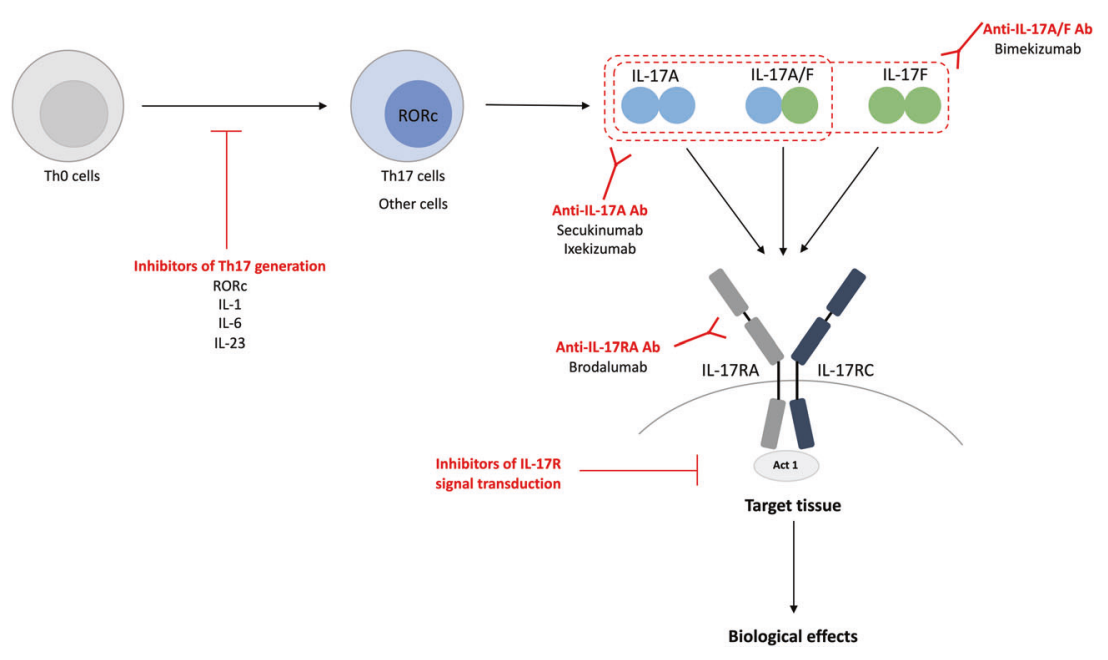

Fig. 3 Options for directly and indirectly targeting IL-17. Direct options to target IL-17 include targeting the IL-17A homodimer and IF-17A/F heterodimer with an anti-IL-17A monoclonal Ab and targeting the IL-17RA chain of the IL-17 receptor with a monoclonal anti-IL-17RA Ab. Another option is targeting both IL-17A and IL-17F with a bispecific Ab. Indirect options to target IL-17 include the use of inhibitors of Th17 cell differentiation with anti-IL-1, anti-IL-6, and anti-IL-23 Abs. Another option is the use of small molecules to target Roryt/Rorc

applies to other chemokines in monocytes, dendritic cells, and Th17 cells involved in the migration of these cells to the liver, such as MCP1 and CCL-20. ${ }^{22}$ Such migration induces hepatitis and liver damage.

Muscles are an important target of these cytokines. In the other cell types described above, in combination with IL-1 or TNF, IL-17 specifically increases the production of IL-6, leading to myocyte inflammation, and in combination with IL-8 or CCL-20, IL-17 leads to neutrophil or Th17 cell migration, respectively. ${ }^{54}$ These effects on heart muscle or the myocardium have obvious importance. Combination of the same cytokines induces defects in cardiomyocyte contractility. ${ }^{54}$ In addition, proinflammatory cytokines, specifically in combination with IL-17, affect cardiac rhythm, leading to arrhythmia and possibly sudden death. ${ }^{55}$

IL-17 production, Th17 differentiation, and the Th17/Treg balance are influenced by many factors, which may contribute to the appearance and severity of chronic inflammatory diseases, including arthritis. One such factor is the microbiota. Segmented filamentous bacteria was found to induce IL-17-driven arthritis in mouse models. ${ }^{56}$ Another factor is a high-salt diet due to its direct cardiovascular effects. A high-salt diet alone was found to shift the balance towards Th17 cells in mouse models. ${ }^{57}$ The same concept was later applied to human RA, in which a high-salt diet was found to increase the effect of smoking on RA incidence and severity. ${ }^{58}$

\section{IL-17-TARGETED THERAPIES}

The contributions of IL-17 and Th17 cells to many diseases are the basis of their clinical targeting. ${ }^{6}$ Many options are possible, and products involving some are already on the market (Fig. 3). The most direct method to target IL-17 is the use of antibodies against IL-17A, such as secukinumab and ixekizumab. ${ }^{3}$ The other option is to block IL-17RA, which is also part of the IL-17E receptor, with brodalumab. Bispecific antibodies against IL-17A and IL-17F, such as bimekizumab, and against IL-17A and TNFa are under clinical development. $^{59}$

Secukinumab and ixekizumab are registered for the treatment of psoriasis, psoriatic arthritis, and ankylosing spondylitis, and brodalumab is registered for the treatment of psoriasis. ${ }^{60}$ These drugs are not registered for RA, as trials showed a high heterogeneity in the degree of responses, in part explained by different genetic backgrounds. ${ }^{61}$

Some products that indirectly target IL-17 are already on the market, while others are in progress. These include affecting Th17 cell differentiation with inhibitors of IL-6, IL-1, or IL-23. Targeting RORc with small molecules or the administration of IL-2 are other possibilities for restoring the Th17/Treg balance. ${ }^{41}$

These treatments have been rather well tolerated so far. Since IL-17 and Th17 cells are critical for host protection against extracellular bacterial and fungal pathogens, an increased frequency of these infections was observed, but this effect did not reach the severity seen in patients with genetic defects in the IL-17 pathway. ${ }^{62}$ A lack of control of Candida has been proposed to explain increased pathogenic activity in Crohn's disease following IL-17 inhibition. ${ }^{63}$

In addition to psoriasis, psoriatic arthritis, and ankylosing spondylitis, clinical trials are ongoing or planned in other diseases, including skin diseases such atopic dermatitis, discoid lupus, pyoderma gangrenosum, and hidradenitis suppurativa and systemic diseases, such as giant cell arteritis, lupus nephritis, non-alcoholic fatty liver disease, type 1 diabetes, and very recently COVID-19.

A key question is the selection of patients with IL-17-driven disease. ${ }^{27}$ As for any treatment, highly heterogeneous responses are observed. ${ }^{60,61}$ As IL-17 acts more as a regulatory cytokine, its circulating protein levels are very low compared to those of, for instance, IL-6. ${ }^{64}$ In addition, IL-17 acts as an amplification factor for other inflammatory cytokines. As discussed above, the function of IL-17 results from the combination of its positive effects with those of IL-1, TNF, and other cytokines, and inhibitory effects with those of IL-25, autoantibodies, and soluble receptors. ${ }^{28}$ The use of bioassays may be a more reliable way to evaluate the function of circulating IL-17. ${ }^{64}$ Such an assay showed a link between RA activity and joint destruction at the early phase of myocardial infarction. ${ }^{52}$ Other options to manipulate the contributions of IL-17 and Th17 cells include changing the microbiota or a low-salt diet. ${ }^{57,58}$

\section{CONCLUSION}

Over the last 25 years, IL-17 has moved from a newly discovered cytokine to a newly registered treatment for inflammatory diseases. ${ }^{6}$ Our understanding of IL-17 has expanded from its local to its systemic effects. As seen from its clinical expression in acute and chronic diseases, the role of IL-17 is regulated through interactions between soluble factors and cells. A key issue that remains to be clarified for improved targeting is an understanding of its heterogeneous contribution to diseases. ${ }^{61}$ This is critical for selecting patients with IL-17-driven disease. ${ }^{64}$ Such personalized 
medicine may lead to an improved response to IL-17-targeted treatment. $^{60}$ New methods to increase these options with different modes of action are under development.

\section{ADDITIONAL INFORMATION}

Conference: This review is based on a talk given during the 17th International Congress of Immunology that took place on 19-23 October 2019 in Beijing, China.

\section{REFERENCES}

1. Yao, Z. et al. Herpesvirus Saimiri encodes a new cytokine, IL-17, which binds to a novel cytokine receptor. Immunity 3, 811-821 (1995).

2. Fossiez, F. et al. T cell interleukin-17 induces stromal cells to produce proinflammatory and hematopoietic cytokines. J. Exp. Med. 183, 2593-2603 (1996).

3. Hueber, W. et al. Effects of AIN457, a fully human antibody to interleukin-17A, on psoriasis, rheumatoid arthritis, and uveitis. Sci. Transl. Med. 2, 52 ra72 (2010).

4. Harrington, L. E. et al. Interleukin 17-producing CD4+ effector T cells develop via a lineage distinct from the $T$ helper type 1 and 2 lineages. Nat. Immunol. 6, 1123-1132 (2005).

5. Park, $H$. et al. A distinct lineage of CD4 T cells regulates tissue inflammation by producing interleukin 17. Nat. Immunol. 6, 1133-1141 (2005).

6. Beringer, A., Noack, M. \& Miossec, P. IL-17 in chronic inflammation: from discovery to targeting. Trends Mol. Med. 22, 230-241 (2016).

7. Rouvier, E., Luciani, M. F., Mattei, M. G., Denizot, F. \& Golstein, P. CTLA-8, cloned from an activated $\mathrm{T}$ cell, bearing AU-rich messenger RNA instability sequences, and homologous to a herpesvirus saimiri gene. J. Immunol. 150, 5445-5456 (1993).

8. Chabaud, M., Fossiez, F., Taupin, J. L. \& Miossec, P. Enhancing effect of IL-17 on IL-1induced IL- 6 and leukemia inhibitory factor production by rheumatoid arthritis synoviocytes and its regulation by Th2 cytokines. J. Immunol. 161, 409-414 (1998).

9. Yao, Z. et al. Human IL-17: a novel cytokine derived from T cells. J. Immunol. 155, 5483-5486 (1995).

10. Pappu, R., Ramirez-Carrozzi, V. \& Sambandam, A. The interleukin-17 cytokine family: critical players in host defence and inflammatory diseases. Immunology 134, 8-16 (2011).

11. McAllister, F. et al. Role of IL-17A, IL-17F, and the IL-17 receptor in regulating growth-related oncogene-alpha and granulocyte colony-stimulating factor in bronchial epithelium: implications for airway inflammation in cystic fibrosis. J. Immunol. 175, 404-412 (2005).

12. Zrioual, S. et al. Genome-wide comparison between IL-17A- and IL-17F-induced effects in human rheumatoid arthritis synoviocytes. J. Immunol. 182, 3112-3120 (2009).

13. Lavocat, F., Ndongo-Thiam, N. \& Miossec, P. Interleukin-25 produced by synoviocytes has anti-inflammatory effects by acting as a receptor antagonist for interleukin-17A function. Front Immunol. 8, 647 (2017).

14. Kotake, S. et al. IL-17 in synovial fluids from patients with rheumatoid arthritis is a potent stimulator of osteoclastogenesis. J. Clin. Investig. 103, 1345-1352 (1999).

15. Chabaud, M. et al. Human interleukin-17: a T cell-derived proinflammatory cytokine produced by the rheumatoid synovium. Arthritis Rheum. 42, 963-970 (1999).

16. van Nieuwenhuijze, A. E. et al. Complementary action of granulocyte macrophage colony-stimulating factor and interleukin-17A induces interleukin-23, receptor activator of nuclear factor-kappaB ligand, and matrix metalloproteinases and drives bone and cartilage pathology in experimental arthritis: rationale for combination therapy in rheumatoid arthritis. Arthritis Res. Ther. 17, 163 (2015).

17. Page, G. et al. Plasma cell-like morphology of Th1-cytokine-producing cells associated with the loss of CD3 expression. Am. J. Pathol. 164, 409-417 (2004).

18. $\mathrm{Li}, \mathrm{H}$. et al. Cloning and characterization of IL-17B and IL-17C, two new members of the IL-17 cytokine family. Proc. Natl Acad. Sci. USA 97, 773-778 (2000).

19. Toy, D. et al. Cutting edge: interleukin 17 signals through a heteromeric receptor complex. J. Immunol. 177, 36-39 (2006).

20. Li, X., Bechara, R., Zhao, J., McGeachy, M. J. \& Gaffen, S. L. IL-17 receptor-based signaling and implications for disease. Nat. Immunol. 20, 1594-1602 (2019).

21. Ely, L. K., Fischer, S. \& Garcia, K. C. Structural basis of receptor sharing by interleukin 17 cytokines. Nat. Immunol. 10, 1245-1251 (2009).

22. Beringer, A., Thiam, N., Molle, J., Bartosch, B. \& Miossec, P. Synergistic effect of interleukin-17 and tumour necrosis factor-alpha on inflammatory response in hepatocytes through interleukin-6-dependent and independent pathways. Clin. Exp. Immunol. 193, 221-233 (2018).

23. Chabaud, M., Page, G. \& Miossec, P. Enhancing effect of IL-1, IL-17, and TNF-alpha on macrophage inflammatory protein-3alpha production in rheumatoid arthritis: regulation by soluble receptors and Th2 cytokines. J. Immunol. 167, 6015-6020 (2001).

24. Herjan, T. et al. IL-17-receptor-associated adaptor Act1 directly stabilizes mRNAs to mediate IL-17 inflammatory signaling. Nat. Immunol. 19, 354-365 (2018).
25. Slowikowski, K. et al. CUX1 and IkappaBzeta (NFKBIZ) mediate the synergistic inflammatory response to TNF and IL-17A in stromal fibroblasts. Proc. Natl Acad. Sci. USA 117, 5532-5541 (2020).

26. Granet, C., Maslinski, W. \& Miossec, P. Increased AP-1 and NF-kappaB activation and recruitment with the combination of the proinflammatory cytokines IL1 beta, tumor necrosis factor alpha and IL-17 in rheumatoid synoviocytes. Arthritis Res. Ther. 6, R190-R198 (2004).

27. Robert, M. \& Miossec, P. IL-17 in rheumatoid arthritis and precision medicine: from synovitis expression to circulating bioactive levels. Front. Med. 5, 364 (2018).

28. Ndongo-Thiam, N., Clement, A., Pin, J. J., Razanajaona-Doll, D. \& Miossec, P. Negative association between autoantibodies against IL-17, IL-17/anti-IL-17 antibody immune complexes and destruction in rheumatoid arthritis. Ann. Rheum. Dis. 75, 1420-1422 (2016).

29. Dakin, S. G. et al. Pathogenic stromal cells as therapeutic targets in joint inflammation. Nat. Rev. Rheumatol. 14, 714-726 (2018).

30. Albanesi, C. et al. Interleukin-17 is produced by both Th1 and Th2 lymphocytes, and modulates interferon-gamma- and interleukin-4-induced activation of human keratinocytes. J. Investig. Dermatol. 115, 81-87 (2000).

31. Aarvak, T., Chabaud, M., Miossec, P. \& Natvig, J. B. IL-17 is produced by some proinflammatory Th1/Th0 cells but not by Th2 cells. J. Immunol. 162, 1246-1251 (1999).

32. Ivanov, I. I. et al. The orphan nuclear receptor RORgammat directs the differentiation program of proinflammatory IL-17+ T helper cells. Cell 126, 1121-1133 (2006).

33. Cua, D. J. \& Tato, C. M. Innate IL-17-producing cells: the sentinels of the immune system. Nat. Rev. Immunol. 10, 479-489 (2010).

34. Noordenbos, T. et al. Human mast cells capture, store, and release bioactive, exogenous IL-17A. J. Leukoc. Biol. 100, 453-462 (2016).

35. Lin, A. M. et al. Mast cells and neutrophils release IL-17 through extracellular trap formation in psoriasis. J. Immunol. 187, 490-500 (2011).

36. Yosef, N. et al. Dynamic regulatory network controlling TH17 cell differentiation. Nature 496, 461-468 (2013)

37. Oppmann, B. et al. Novel p19 protein engages IL-12p40 to form a cytokine, IL-23, with biological activities similar as well as distinct from IL-12. Immunity 13, 715-725 (2000).

38. Aggarwal, S., Ghilardi, N., Xie, M. H., de Sauvage, F. J. \& Gurney, A. L. Interleukin-23 promotes a distinct CD4 T cell activation state characterized by the production of interleukin-17. J. Biol. Chem. 278, 1910-1914 (2003).

39. Murphy, C. A. et al. Divergent pro- and antiinflammatory roles for IL-23 and IL-12 in joint autoimmune inflammation. J. Exp. Med. 198, 1951-1957 (2003).

40. Lee, Y. et al. Induction and molecular signature of pathogenic TH17 cells. Nat. Immunol. 13, 991-999 (2012).

41. Noack, M. \& Miossec, P. Th17 and regulatory T cell balance in autoimmune and inflammatory diseases. Autoimmun. Rev. 13, 668-677 (2014).

42. DuPage, M. \& Bluestone, J. A. Harnessing the plasticity of CD4(+) T cells to treat immune-mediated disease. Nat. Rev. Immunol. 16, 149-163 (2016).

43. Noack, M., Ndongo-Thiam, N. \& Miossec, P. Interaction among activated lymphocytes and mesenchymal cells through podoplanin is critical for a high IL-17 secretion. Arthritis Res. Ther. 18, 148 (2016).

44. Noack, M., Ndongo-Thiam, N. \& Miossec, P. Role of podoplanin in the high interleukin-17A secretion resulting from interactions between activated lymphocytes and psoriatic skin-derived mesenchymal cells. Clin. Exp. Immunol. 186, 64-74 (2016).

45. Sato, K. et al. Th17 functions as an osteoclastogenic helper T cell subset that links T cell activation and bone destruction. J. Exp. Med. 203, 2673-2682 (2006).

46. Chabaud, M. \& Miossec, P. The combination of tumor necrosis factor alpha blockade with interleukin-1 and interleukin-17 blockade is more effective for controlling synovial inflammation and bone resorption in an ex vivo model. Arthritis Rheum. 44, 1293-1303 (2001).

47. Osta, B. et al. Differential effects of IL-17A and TNF-alpha on osteoblastic differentiation of isolated synoviocytes and on bone explants from arthritis patients. Front Immunol. 6, 151 (2015)

48. Feldmann, M. \& Maini, R. N. Anti-TNF alpha therapy of rheumatoid arthritis: what have we learned? Annu Rev. Immunol. 19, 163-196 (2001).

49. Libby, P., Ridker, P. M. \& Hansson, G. K. Progress and challenges in translating the biology of atherosclerosis. Nature 473, 317-325 (2011).

50. Beringer, A. \& Miossec, P. Systemic effects of IL-17 in inflammatory arthritis. Nat. Rev. Rheumatol. 15, 491-501 (2019).

51. Hot, A., Lenief, V. \& Miossec, P. Combination of IL-17 and TNFalpha induces a proinflammatory, pro-coagulant and pro-thrombotic phenotype in human endothelial cells. Ann. Rheum. Dis. 71, 768-776 (2012).

52. Bochaton, T. et al. Early kinetics of serum Interleukine-17A and infarct size in patients with reperfused acute ST-elevated myocardial infarction. PLOS One 12, e0188202 (2017). 
53. Libby, P. \& Ridker, P. M. Inflammation and atherosclerosis: role of C-reactive protein in risk assessment. Am. J. Med. 116, 9S-16S (2004).

54. Beringer, A., Gouriou, Y., Lavocat, F., Ovize, M. \& Miossec, P. Blockade of storeoperated calcium entry reduces IL-17/TNF cytokine-induced inflammatory response in human myoblasts. Front. Immunol. 9, 3170 (2018).

55. Lazzerini, P. E., Laghi-Pasini, F., Boutjdir, M. \& Capecchi, P. L. Commentary: systemic effects of IL-17 in inflammatory arthritis. Front. Cardiovasc Med. 6, 183 (2019).

56. $\mathrm{Wu}, \mathrm{H}$. J. et al. Gut-residing segmented filamentous bacteria drive autoimmune arthritis via T helper 17 cells. Immunity 32, 815-827 (2010).

57. Wu, C. et al. Induction of pathogenic TH17 cells by inducible salt-sensing kinase SGK1. Nature 496, 513-517 (2013).

58. Jiang, X. et al. High sodium chloride consumption enhances the effects of smoking but does not interact with SGK1 polymorphisms in the development of ACPA-positive status in patients with RA. Ann. Rheum. Dis. 75, 943-946 (2016).

59. Glatt, S. et al. Dual IL-17A and IL-17F neutralisation by bimekizumab in psoriatic arthritis: evidence from preclinical experiments and a randomised placebo- controlled clinical trial that IL-17F contributes to human chronic tissue inflammation. Ann. Rheum. Dis. 77, 523-532 (2018).

60. Baker, K. F. \& Isaacs, J. D. Novel therapies for immune-mediated inflammatory diseases: What can we learn from their use in rheumatoid arthritis, spondyloarthritis, systemic lupus erythematosus, psoriasis, Crohn's disease and ulcerative colitis? Ann. Rheum. Dis. 77, 175-187 (2018).

61. Burmester, G. R. et al. Association of HLA-DRB1 alleles with clinical responses to the anti-interleukin-17A monoclonal antibody secukinumab in active rheumatoid arthritis. Rheumatology 55, 49-55 (2016).

62. Puel, A. et al. Inborn errors of human IL-17 immunity underlie chronic mucocutaneous candidiasis. Curr. Opin. Allergy Clin. Immunol. 12, 616-622 (2012).

63. Colombel, J. F., Sendid, B., Jouault, T. \& Poulain, D. Secukinumab failure in Crohn's disease: the yeast connection? Gut 62, 800-801 (2013).

64. Ndongo-Thiam, N. \& Miossec, P. A cell-based bioassay for circulating bioactive IL17: application to destruction in rheumatoid arthritis. Ann. Rheum. Dis. 74, 1629-1631 (2015). 